\title{
In vitro susceptibility of Mycobacterium tuberculosis to clarithromycin
}

\author{
Mojtaba Varshochi ${ }^{1 \star}$, Hossein Najafian², Seyed Reza Moaddab ${ }^{2}$ and Behrooz Nagili ${ }^{1}$ \\ ${ }^{1}$ Infectious Diseases and Tropical Medicine Research Center, Tabriz University of Medical Sciences, Tabriz, Iran. \\ ${ }^{2}$ Faculty of Medicine, Tabriz University of Medical Sciences, Tabriz, Iran.
}

Accepted 30 October, 2012

\begin{abstract}
This study was done to evaluate in vitro susceptibility of Mycobacterium tuberculosis of clarithromycin. In this prospective study, $90 \mathrm{M}$. tuberculosis strains (40 resistant strains and 50 susceptible strains) referred to the Tabriz laboratory of TB research Center were selected and cultured in L-J medium and with 3 doses of clarithromycin. $\mathbf{4 0}$ strains were resistant and $\mathbf{5 0}$ strains were sensitive to first line drugs. $60 \%$ of strains were resistant to clarithromycin with $20 \mu \mathrm{g} / \mathrm{ml}$. Significant difference was not found if resistant rate of $M$. tuberculosis strains to clarithromycin with 20 and $40 \mu \mathrm{g} / \mathrm{ml}$ and all studied strain was sensitive to clarithromycin with $80 \mu \mathrm{g} / \mathrm{ml}$. 33 strains of resistant strains and 48 strains of sensitive strains to first anti tuberculosis line drug was sensitive to clarithromycin with $40 \mu \mathrm{g} / \mathrm{ml}$. Based on the results of this study and other studies, the need for extensive laboratory study and using liquid culture (including Midelbary) and advanced techniques such as BACTEC and even more animal models are necessary. The long-term clinical study using clarithromycin in combination with anti-TB therapy, especially in the case of MDR-TB strains is necessary for the low side effect as clarithromycin in the treatment of tuberculosis, in the form of suitable compounds which can be used as second drug.
\end{abstract}

Key words: Mycobacterium tuberculosis, drug resistant, clarithromycin, strains.

\section{INTRODUCTION}

Re-incidence of infection and disease caused by Mycobacterium tuberculosis and the increasing prevalence of multi-drug resistance among strains of $M$. tuberculosis evokes the idea that new drugs are still needed (Brennan and Nikaido, 1995). Among the candidates for second-line treatment of Tuberculosis of the macrolides, clarithromycin can be named. While mycobacterial cell membrane acts as a barrier impermeable to the medication, they penetrate the mycobacterial cell membrane and influence them (Bermudez et al., 1998).

Clinical experience with macrolides antibiotics for $M$. tuberculosis is insufficient, thus the necessity of applying combinations of several drugs in the regimen therapy doubles the need for prior assessments of individual impact of Clarithromycin. Whether clarithromycin is effective

*Corresponding author. E-mail: varshochim@yahoo.com. Tel: +98-9143135846. on patients or not was examined by measuring its effect on the improvement of colony forming units (CFU) of $M$. tuberculosis from sputum of patients with smear-positive pulmonary Tuberculosis (TB) (Mitnick et al., 2008).

Increasing incidence of resistant tuberculosis and increasing resistance to anti-mycobacterial drugs, especially in patients infected with human immunodeficiency virus (HIV), points out the immediate need for new compounds to overcome the resistance. Reassessment of old compounds such as macrolides, has been widely spread, which can be a way to deal with this problem (Sato and Tomioka, 1999a; Brudney and Dobkin, 1992). Systematic study of macrolides and matching it with data on the susceptibility of $M$. tuberculosis to antibiotics in this group could be noteworthy in the study on effective compounds (Bosne-David et al., 2000).

These results are consistent with other genetic and phenotypic studies showing the least heterogeneity in $M$. tuberculosis (Bermudez et al., 1998). The aim of this study was to evaluate drug susceptibility of $M$. tuberculosis to clarithromycin in in vitro condition. 


\section{MATERIALS AND METHODS}

This study was prospectively conducted on 90 isolated strains of $M$. tuberculosis from patients at TB Research center of Tabriz, 50 of which were susceptible to the drug of first-line treatment of tuberculosis and 40 of which were resistance to the drug of first-line treatment of tuberculosis. These strains were randomly selected from among patients referred to the laboratory of TB Research Center of Tabriz. Resistant strains were selected from among hundreds of strains isolated during recent years in the Laboratory of TB Research Center.

Antibiogram was conducted with proportional method in Lowenstein-Jensen environment. Three doses of clarithromycin were used to determine the susceptibility and resistance. For internal quality control in laboratory, for some strains, the Middlebrook 7 $\mathrm{H} 9$ agar environment and disk diffusion method were also used. For this purpose, strains susceptible and resistant to drugs of first-line treatment of tuberculosis were cultured in the Lowenstein-Jensen medium; then 3 different doses of clarithromycin were used to determine the susceptibility and measure the minimum inhibitory concentrations (MIC). Drug resistance was considered as bacterial growth over $1 \%$ in medium containing antibiotic compared to the drug-free medium.

In this study, susceptibility tests and MIC determination have been conducted by agar dilution on $7 \mathrm{H} 10$ agar enriched with the oleic acid, albumin, dextrose and catalase. The Mycobacterium strains were grown in $7 \mathrm{H} 9$ broth at $37^{\circ} \mathrm{C}$ and diluted with distilled water for compliance with the Mc-Farland standard turbidity. The results were read after 3 weeks of incubation at a pressure of 1 atmosphere of $\mathrm{CO}_{2}$ at $37^{\circ} \mathrm{C}$; MIC is defined as the minimum concentration of antibiotic completely inhibiting visible bacterial growth (a colony was not considered). Antibiotics under study, each of which prepared by manufacturers as standard powders, the stability of antibiotics during the incubation period with the Petri was monitored by $E$. coli control species at days 1 and 11 .

The samples were then evaluated by experts in TB Research Center of Tabriz; due to contamination in some samples, the tests were repeated and the final results, after tests accuracy confirmation, were determined as the susceptibility and resistance to different doses, and the MIC were then calculated for each of the resistant strains; then, the obtained data and drug resistance history of each strain was determined based on archive of research center, and supplementary information, including gender of the patients. The obtained results were then analyzed by SPSS 11.5 software and Chi -square statistical test, and the significance level was considered as $\mathrm{P}<0.05$.

\section{RESULTS}

Susceptibility and resistance of $M$. tuberculosis strains (MTS) to several dosage of clarithromycin between two groups of strains have been shown in Table 1. There was no significant difference in the rates of resistance and susceptibility to clarithromycin with doses of $20 \mu \mathrm{g} / \mathrm{ml}(\mathrm{P}$ $=0.083)$ and $40 \mu \mathrm{g} / \mathrm{ml}(P=0.072)$ between strain groups (strains resistant to first-line anti-TB drugs and strains susceptible to first-line anti-TB drugs) and all strains under study were susceptible to $80 \mu \mathrm{g} / \mathrm{ml}$ dose of clarithromycin (Table 1). Susceptibility and resistance of $M$. tuberculosis strains (MTS) to first-line anti-TB drugs have been shown in Table 2. There was no significant difference in susceptibility or resistance to clarithromycin among strains with different number of resistance to the first generation of drugs $(P=0.052)$. Susceptibility and resistance of $M$. tuberculosis strains (MTS) to first-line anti-TB drugs and several doses of clarithromycin have been shown in Table 3.

\section{DISCUSSION}

M. tuberculosis remains a leading cause of mortalities associated with infectious disease agents in the world. In 1997, about 2 billion people were estimated to be infected with tuberculosis (Brennan and Nikaido, 1995). Every year, 8 to 9 million people get infected with TB and finally, 2 million people die of TB or symptoms of infection (Brennan and Nikaido, 1995). TB is a major problem in developing countries so that $95 \%$ of patients and $90 \%$ of mortalities due to this disease occur in these countries. In African regions, where the prevalence of infection with HIV is more than the most areas of the world, TB mortality rate is over $50 \%$ (Brennan and Nikaido, 1995).

In 1993, World Health Organization (WHO) called tuberculosis a global public health emergency problem. Since then on, TB and related mortality have remained constant. Despite the relative success in this case, we have been witnessing an increasing drug resistance; so that this phenomenon is now considered a global threat. Resistance to an anti-tuberculosis drug has been recognized many years ago. Unfortunately, the evolution of drug resistance in TB treatment has led to strains resistant to multiple drugs (MDR-TB: Multi Drug Resistant-TB).

The incidence of MDR-TB is increasing rapidly and the estimated incidence in the whole world was 460,000 cases in 2005 (Bermudez et al., 1998). Due to lack of appropriate diagnostic and laboratory facilities and sometimes lack of adequate and reliable reporting system, the prevalence of MDR-TB in many developing countries is estimated lower than the actual rate. Almost two third of MDR-TB cases are seen in the three countries; Russia, China and India.

In a study in Iran, sputum samples were taken from 2,030 patients with known TB and examined for $M$. bacilli (AFB) by microscopic examination and were cultured in Lowenstein-Jensen standard medium. M. tuberculosis was susceptible to first-line drugs in 1,284 and then MDR-TB was observed in 113 patients. Totally, 12 (about $10 \%$ ) of MDR-TB cases were resistant to all second-line TB treatment drugs which are called XDR-TB (Extensively Drug Resistant TB). In the recent study, two groups of XDR-TB are described (Sato and Tomioka, 1999b). If these over-resistant XDR-TB pathogens find a chance of growth and reproduction, we will encounter a serious global health and economic problem.

Insignificant available experience and the results obtained from previous studies indicate that antibiotics of Macrolides family are effective against $M$. tuberculosis in patients with TB disease, from among which, 
Table 1. Susceptible and resistant Mycobacterium tuberculosis strains (MTS) to several dosage of clarithromycin between two groups of strains.

\begin{tabular}{llccl}
\hline \multirow{2}{*}{ Parameter $(\mu \mathrm{g} / \mathrm{ml})$} & \multicolumn{3}{c}{ Group } & \multirow{2}{*}{$\mathbf{P}$} \\
\cline { 3 - 4 } & & Resistant & Susceptible & \\
\hline \multirow{2}{*}{ Clarithromycin 20 } & Resistant & 20 & 34 & \multirow{2}{*}{0.083} \\
& Susceptible & 20 & 16 & \\
\cline { 3 - 4 } Clarithromycin 40 & Resistant & 7 & 2 & \multirow{2}{*}{0.072} \\
& Susceptible & 33 & 48 & \\
Clarithromycin 80 & Susceptible & 40 & 50 & - \\
\hline
\end{tabular}

Table 2. Susceptible and resistant Mycobacterium tuberculosis strains (MTS) to first-line anti-TB drugs.

\begin{tabular}{lcc}
\hline \multirow{2}{*}{ Parameter } & Susceptible and resistant of Mycobacterium tuberculosis strains \\
\cline { 2 - 3 } & Resistant & Susceptible \\
\hline Isoniazid & 20 & 70 \\
Rifampin & 9 & 81 \\
Streptomycin & 28 & 62 \\
Ethambutol & 5 & 85 \\
\hline
\end{tabular}

Table 3. Susceptible and resistant Mycobacterium tuberculosis strains (MTS) to first-line anti-TB drugs and several doses of clarithromycin.

\begin{tabular}{|c|c|c|c|c|c|c|c|c|c|}
\hline \multirow{2}{*}{ Parameter } & & \multicolumn{2}{|c|}{ Clarithromycin $20(\mu \mathrm{g} / \mathrm{ml})$} & \multirow{2}{*}{$\mathbf{P}$} & \multicolumn{2}{|c|}{ Clarithromycin $40(\mu \mathrm{g} / \mathrm{ml})$} & \multirow{2}{*}{$\mathbf{P}$} & \multirow{2}{*}{$\begin{array}{c}\text { Clarithromycin } 80(\mu \mathrm{g} / \mathrm{ml}) \\
\text { Susceptible }\end{array}$} & \multirow{2}{*}{$\mathbf{P}$} \\
\hline & & Resistant & Susceptible & & Resistant & Susceptible & & & \\
\hline \multirow{2}{*}{ Isoniazid } & Resistant & 5 & 15 & \multirow{2}{*}{$<0.001$} & 2 & 18 & \multirow{2}{*}{0.682} & 20 & \multirow{2}{*}{ - } \\
\hline & Susceptible & 49 & 21 & & 7 & 63 & & 70 & \\
\hline \multirow{2}{*}{ Rifampin } & Resistant & 3 & 6 & \multirow{2}{*}{0.148} & 3 & 6 & \multirow{2}{*}{0.043} & 9 & \multirow{2}{*}{ - } \\
\hline & Susceptible & 51 & 30 & & 6 & 75 & & 81 & \\
\hline \multirow{2}{*}{ streptomycin } & Resistant & 14 & 14 & \multirow{2}{*}{0.247} & 3 & 25 & \multirow{2}{*}{0.574} & 28 & \multirow{2}{*}{ - } \\
\hline & Susceptible & 40 & 22 & & 6 & 56 & & 62 & \\
\hline \multirow{2}{*}{ Ethambutol } & Resistant & 3 & 2 & \multirow{2}{*}{0.669} & 1 & 4 & \multirow{2}{*}{0.417} & 5 & \multirow{2}{*}{ - } \\
\hline & Susceptible & 51 & 34 & & 8 & 77 & & 85 & \\
\hline
\end{tabular}

clarithromycin was more favored and tested due to three reasons:

1. Biochemical drug and susceptibility predicted the activity rate;

2. Clinical findings, although incomprehensive, indicated the effectiveness of this drug and;

3. This drug can be administered orally and its bactericidal effect is fast.

For the first time, these drugs were evaluated by
Mitchison while categorizing effectiveness of anti-TB drugs. After making sure of the relationship between the effectiveness of drugs in both in vitro and in vivo (inside the body) conditions proved in a drug class, it can be extended to other substances showing a similar activity in the laboratory (Brennan and Nikaido, 1995).

In a study by Parsons et al. (1997) at infectious diseases section of the Wadsworth Center in New York, they stated that drug resistance to anti-tuberculosis drugs is a global problem and affirmed that new drugs effective on M. tuberculosis are required. In a study by Mor and 
Esfandiari (1997) at the University of California, United States, examining the combined effect of clarithromycin and pyrazinamide on M. tuberculosis, they stated that this combination has a synergistic effect on $M$. tuberculosis. In a study by Cavalieri et al. (1995) at the University of Omaha, United States, they stated that clarithromycin in combination with other anti-TB drugs has good effect on strains of $M$. tuberculosis. In a study by Sato and Tomioka (1999a) at the Department of Microbiology, University of Izumo, Japan, examining the effect of clarithromycin on $M$. tuberculosis strains, they stated that clarithromycin is effective on $M$. tuberculosis strains and M. tuberculosis is susceptible to clarithromycin (Sato and Tomioka, 1999a).

In a study by Tomioka et al. (2000) at the Department of Microbiology, University of Izumo, Japan, they stated that clarithromycin was effective on $M$. tuberculosis strains and $M$. tuberculosis is susceptible to this antibiotic (Tomioka et al., 2000). In our study, M. tuberculosis strains were susceptible to clarithromycin with doses of 20,40 and $80 \mu \mathrm{g} / \mathrm{ml}$ at 40,90 and $100 \%$ of cases. In a study by Bhusal et al. (2005) at the University of Ryukyus in Okinawa, Japan, with examining the effect of the clarithromycin on $M$. tuberculosis, they expressed that the antibiotic under their study has synergistic effect on this micro-organisms along with other anti-TB antibiotics.

Macrolides are one of the most important antibiotics for human use. This group of drugs is widely used in medicine due to their wide range of activity and few side effects. Experimental studies have shown that macrolides have anti-mycobacterial activity and their combinations with Ethambutol (one of the first-line anti-TB drugs) may increase its effectiveness on Tuberculosis bacilli (Brennan and Nikaido, 1995). The results suggest that both drugs should be investigated further as potential adjuncts to the treatment of resistant tuberculosis, in particular through new drug delivery systems such as the dry powder inhaler allowing high lung deposition (Umubyeyi et al., 2007; Stoffels et al., 2009).

For clofazimine, amikacin, clarithromycin, ciprofloxacin, and amoxicillin/clavulanate, all the MIC (90) of the MDR isolates exceeded those of non-MDR isolates (Lu et al., 2008). Cremades et al., (2008) demonstrated that addition of clarithromycin to anti-tuberculosis regimens in MDR was beneficial.

In addition, the clinical findings suggest that betalactamase have good future for use in treating patients with MDR-TB. However, more studies are needed to determine the interaction of these drugs with first line anti-TB drugs.

\section{Conclusion}

According to the study on the effects of clarithromycin on strains of $M$. tuberculosis susceptible and resistant to first line anti-TB drugs, in both Lowenstein-Jensen environment and in vitro, respectively, 60 and $10 \%$ of strains of $M$. tuberculosis were resistant to clarithromycin with doses of 20 and $40 \mu \mathrm{g} / \mathrm{ml}$. There was no significant difference in the resistance of $M$. tuberculosis to clarithromycin with doses of 20 and $40 \mu \mathrm{g} / \mathrm{ml}$; while all strains of $M$. tuberculosis (susceptible and resistant to first-line anti-TB drugs) were susceptible to clarithromycin with dose of 80 $\mu \mathrm{g} / \mathrm{ml}$. There was no significant difference between susceptible and resistant strains, and even the noninterference of the number of resistance to the firstline anti-tuberculosis drugs in the susceptibility to different doses of clarithromycin show that clarithromycin might be a good option in combined treatment of resistant TB cases. We have tested three different concentrations of clarithromycin to set a break point for clarithromycin, a greater number of different concentrations of clarithromycin are recommended.

\section{REFERENCES}

Bermudez LE, Petrotsky M, Kolonoki P (1998). Emergency of Mycobacterium avium papulations resistant to macrulides during experimental chemotherapy. Antimicrob. Agents Chemother. 42:180183.

Bhusal Y, Shiohira CM, Yamane N (2005). Determination of in vitro synergy when three antimicrobial agents are combined against Mycobacterium tuberculosis. Int. J. Antimicrob. Agents 26(4):292297.

Bosne-David S, Barros V, Verde SC, Portugal C, David HL (2000). Intrinsic resistance of Mycobacterium tuberculosis to clarithromycin is effectively reversed by subinhibitory concentrations of cell wall inhibitors. J. Antimicrob. Chemother. 46(3):391-395.

Brennan PJ, Nikaido H (1995). The envelope of mycobacteria. Annu. Rev. Biochem. 64:29-63.

Brudney K, Dobkin J (1992). Resurgent tuberculosis in New York City: human immunodeficiency virus, homelessness, and the decline of tuberculosis control programs. Am. Rev. Respir. Dis. 144:745-749.

Cavalieri SJ, Biehle JR, Sanders WE Jr (1995). Synergistic activities of clarithromycin and antituberculous drugs against multidrug-resistant Mycobacterium tuberculosis. Antimicrob. Agents Chemother. 39(7):1542-1545.

Cremades R, Santos A, Rodríguez JC, Garcia-Pachon E, Ruiz M, Royo $G$ (2008). In vitro bactericidal activity of antibiotic combinations against clinical isolates of Mycobacterium chelonae. J. Chemother. 20(1):43-47.

Lu PL, Peng CF, Hwang JJ, Chen YH (2008). Activity of twelve secondline antimicrobial agents against Mycobacterium tuberculosis in Taiwan. J. Chemother. 20(2):202-207.

Mitnick CD, Sonya SS, Kwonjune JS, Michael LR, Atwood SS, Furin JJ, Fitzmaurice GM, Alcantara VFA, Appleton SC, Bayona JN, Bonilla CA, Chalco K, Choi S, Franke MF, Fraser HS, Guerra D, Hurtado RM, Jazayeri D, Joseph K, Llaro K, Mestanza L, Mukherjee JS, Munoz M, Palacios E, Sanchez E, Sloutsky A, Becerra MC (2008). Comprehensive Treatment of Extensively Drug-Resistant Tuberculosis. N. Engl. J. Med. 359:563-574.

Mor N, Esfandiari A (1997). Synergistic activities of clarithromycin and pyrazinamide against Mycobacterium tuberculosis in human macrophages. Antimicrob. Agents Chemother. 41(9):2035-2036.

Parsons LM, Driscoll JR, Taber HW, Salfinger M (1997). Drug resistance in tuberculosis. Infect. Dis. Clin. North. Am. 11(4):905-928.

Sato K, Tomioka H (1999a). Antimicrobial activities of benzoxazinorifamycin (KRM-1648) and clarithromycin against Mycobacterium avium-intracellulare complex within murine peritoneal macrophages, human macrophage-like cells and human alveolar epithelial cells. J. Antimicrob. Chemother. 43(3):351-357.

Sato K, Tomioka H (1999b). In vitro antimicrobial activities of quinolones, 
rifamycins and macrolides against Mycobacterium tuberculosis and $M$. avium complex: attempt to establish new assay methods which accurately reflect therapeutic effects of test agents in vivo. Kekkaku. 74(1):63-70.

Stoffels K, Traore H, Vanderbist F, Fauville-Dufaux M (2009). The effect of combined tobramycin- clarithromycin on Mycobacterium tuberculosis isolates. Int. J. Tuberc. Lung Dis. 13(8):1041-1044.

Tomioka H, Sato K, Kajitani H, Akaki T, Shishido S (2000). Comparative antimicrobial activities of the newly synthesized quinolone WQ-3034, levofloxacin, sparfloxacin, and ciprofloxacin against Mycobacterium tuberculosis and Mycobacterium avium complex. Antimicrob. Agents Chemother. 44(2):283-286.

Umubyeyi A, Rigouts L, Shamputa IC, Dediste A, Struelens M, Portaels $F$ (2007). Low levels of second-line drug resistance among multidrug resistant Mycobacterium tuberculosis isolates from Rwanda. Int. J. Infect. Dis. 12(2):152-156. 